\title{
On a Problem of Cross-Diffusion with Nonlocal Boundary Conditions
}

\author{
Zafar R. Rakhmonov* \\ National University of Uzbekistan \\ University, 4, Tashkent, 100174 \\ Uzbekistan \\ Jasur E. Urunbayev ${ }^{\dagger}$ \\ Samarkand State University \\ University Blvd., 15, Samarkand, 140104 \\ Uzbekistan
}

Received 01.04.2019, received in revised form 15.06.2019, accepted 20.08.2019

Condition of global existence of solution of a non-linear system of cross-diffusion with non-linear boundary conditions is studied in the paper. Critical exponents of Fujita type and critical exponents of global existence of solution are established.

Keywords: nonlinear parabolic system, cross-diffusion, critical Fujita curve, blow-up.

DOI: $10.17516 / 1997-1397-2019-12-5-614-620$.

\section{Introduction and preliminaries}

Let us consider the following nonlinear system of cross-diffusion equations with nonlinear boundary conditions

$$
\begin{gathered}
\frac{\partial u}{\partial t}=\frac{\partial}{\partial x}\left(v^{m_{1}-1} \frac{\partial u}{\partial x}\right), \quad \frac{\partial v}{\partial t}=\frac{\partial}{\partial x}\left(u^{m_{2}-1} \frac{\partial v}{\partial x}\right), \quad x \in R_{+}, \quad t>0, \\
-v^{m_{1}-1} \frac{\partial u}{\partial x}(0, t)=u^{q_{1}}(0, t), \quad-u^{m_{2}-1} \frac{\partial v}{\partial x}(0, t)=v^{q_{2}}(0, t), \quad t>0, \\
u(x, 0)=u_{0}(x), \quad v(x, 0)=v_{0}(x), \quad x \in R_{+},
\end{gathered}
$$

where $m_{i}>1, q_{i}>0(i=1,2), u_{0}$ and $v_{0}(x)$ are non-negative continuous functions with compact support in $R_{+}$.

The process of cross-diffusion means that the spatial displacement of one object, described by one of the variables, occurs due to diffusion of another object described by another variable.

Cross-diffusion models are used in various fields of natural science. For example, in physical systems (plasma physics) [1-3], in chemical systems (dynamics of electrolytic solutions), in biological systems (cross-diffusion transport, dynamics of population), in ecology (dynamics of the forest structure), in seismology - the Burridge-Knopoff model which describes the interaction of tectonic plates [4-7]. In recent years, mathematical models with cross-diffusion have been actively used to study biological populations and the motion of tectonic plates $[4,5]$.

\footnotetext{
*zraxmonov@inbox.ru

†urin1987@rambler.ru

(C) Siberian Federal University. All rights reserved
} 
It is known that a system of degenerate equations may not have a classical solution in the region where $u, v \equiv 0$. In this case, the generalized solution of system (1) is studied in a class where

$$
u(x, t), \quad v(x, t) \geqslant 0, \quad v^{m_{1}-1} \frac{\partial u}{\partial x}, \quad u^{m_{2}-1} \frac{\partial v}{\partial x} \in C(R \times(0,+\infty)),
$$

and system (1) is satisfied from the point of distribution $[1,3]$.

In recent years, the condition for global existence of solutions and the condition for the emergence of a blow-up regime have been intensively studied (see [5-16]). The following system of equations was considered $[8,9]$

$$
\begin{gathered}
u_{t}=u_{x x}, \quad v_{t}=v_{x x}, \quad x>0, \quad 0<T<0, \\
-u_{x}(0, t)=u^{\alpha} v^{p}, \quad-v_{x}(0, t)=u^{q} v^{\beta}, \quad 0<t<T, \\
u(x, 0)=u_{0}(x), \quad v(x, 0)=v_{0}(x), \quad x>0 .
\end{gathered}
$$

The conditions for global solvability in terms of time were studied and the estimation of the solution near explosion time was stated. It was proved that if $p q \leqslant(1-\alpha)(1-\beta)$ then each solution of problem (4)-(6) is global.

The following problem was studied [10]

$$
\begin{gathered}
u_{t}=\left(u^{n}\right)_{x x}, \quad v_{t}=\left(v^{k}\right)_{x x}, \quad x \in R_{+}, \quad t>0, \\
-\left(u^{n}\right)_{x}(0, t)=v^{p}(0, t), \quad-\left(v^{k}\right)_{x}(0, t)=u^{q}(0, t), \quad t>0, \\
u(x, 0)=u_{0}(x), \quad v(x, 0)=v_{0}(x), \quad x \in R_{+} .
\end{gathered}
$$

It was shown that solution of problem $(7)-(9)$ is global if $p q \leqslant(n+1)(k+1) / 4$. Conditions were obtained for the numerical parameters of system $(7)-(9)$ wherein the solution of the problem blows-up in a finite time.

System (7) was considered also with the following boundary conditions [11]

$$
-\left(u^{n}\right)_{x}(0, t)=u^{\alpha} v^{p}(0, t), \quad-\left(v^{k}\right)_{x}(0, t)=u^{q} v^{\beta}(0, t), \quad t>0 .
$$

It was shown that $\min \left\{y_{1}-r_{1}, y_{2}-r_{2}\right\}=0$, where

$$
\begin{aligned}
r_{1}=\frac{2 p+k+1-2 \beta}{4 p q-(k+1-2 \alpha)(n+1-2 \beta)}, & r_{2}=\frac{2 p+n+1-2 \beta}{4 p q-(k+1-2 \alpha)(n+1-2 \beta)}, \\
y_{1}=\frac{1-r_{1}(n-1)}{2}, & y_{2}=\frac{1-r_{2}(k-1)}{2},
\end{aligned}
$$

are critical exponents of Fujita type.

Definition $1[1])$. The solution $\widehat{u}(x, t), \widehat{v}(x, t)$ is called lower solution (upper solution) in $Q_{T}=$ $\left\{(x, t): x \in R_{+}, 0<t<+\infty\right\}$ if $\widehat{u}(x, t)=0$ and $\widehat{v}(x, t)=0$ in $Q_{T} \backslash D_{t}, D_{t}==\{|x|<\rho(t)\} \times$ $(0,+\infty), \widehat{u}(x, t), \widehat{v}(x, t) \in C_{t, x}^{1,2}(D) \cap C\left(Q_{T}\right), \widehat{v}^{m_{1}-1} \frac{\partial \widehat{u}}{\partial x}, \widehat{u}^{m_{2}-1} \frac{\partial \widehat{v}}{\partial x} \in C\left(Q_{T}\right)$ and it satisfies

$$
\begin{gathered}
\frac{\partial \widehat{u}}{\partial t} \leqslant(\geqslant) \frac{\partial}{\partial x}\left(\widehat{v}^{m_{1}-1} \frac{\partial \widehat{u}}{\partial x}\right), \quad \frac{\partial \widehat{v}}{\partial t} \leqslant(\geqslant) \frac{\partial}{\partial x}\left(\widehat{u}^{m_{2}-1} \frac{\partial \widehat{v}}{\partial x}\right), \\
-\widehat{v}^{m_{1}-1} \frac{\partial \widehat{u}}{\partial x}(0, t) \leqslant(\geqslant) \widehat{u}^{q_{1}}(0, t), \quad-\widehat{u}^{m_{2}-1} \frac{\partial \widehat{v}}{\partial x}(0, t) \leqslant(\geqslant) \widehat{v}^{q_{2}}(0, t), \\
\widehat{u}(x, 0) \leqslant(\geqslant) u_{0}(x), \quad \widehat{v}(x, 0) \leqslant(\geqslant) v_{0}(x), \quad x \in R .
\end{gathered}
$$


The purpose of this paper is to find conditions for existence of solutions of problem (1)-(3) in time on the basis of self-similar analysis. Various self-similar solutions of problem (1)-(3) were constructed, the assessment and asymptotic solutions were obtained, critical exponents of Fujita type and critical exponents of global existence of the solution were established.

\section{Main results}

Theorem 2.1. If $q_{1} \leqslant 1, q_{2} \leqslant 1$ then each solution of problem (1)-(3) is global.

Proof. Sufficient conditions for the solvability in time of problem (1)-(3) are determined by constructing its bounded upper solutions. The bounded upper solutions of the problem are taken in the following self-similar form

$$
\left\{\begin{array}{l}
\widehat{u}(x, t)=e^{L_{1} t}\left(K+e^{-M_{1} \xi_{1}}\right), \quad \xi_{1}=x e^{-J_{1} t}, \quad x \geqslant 0, t \geqslant 0 \\
\widehat{v}(x, t)=e^{L_{2} t}\left(K+e^{-M_{2} \xi_{2}}\right), \quad \xi_{2}=x e^{-J_{2} t}, \quad x \geqslant 0, t \geqslant 0
\end{array}\right.
$$

where $K>\max \left\{\left\|u_{0}\right\|_{\infty},\left\|v_{0}\right\|_{\infty}\right\}, \quad M_{1}=(K+1)^{\frac{q_{1}}{m_{1}-1}}, \quad M_{2}=(K+1)^{\frac{q_{2}}{m_{2}-1}}, \quad L_{1}=$ $=\frac{M_{1}(K+1)^{m_{1}-2}\left(M_{1} K+M_{2}\left(m_{1}-1\right)\right)}{K+1}, L_{2}=\frac{M_{2}(K+1)^{m_{2}-2}\left(M_{2} K+M_{1}\left(m_{2}-1\right)\right)}{K+1}, J_{1}=$ $=L_{2}\left(m_{1}-1\right), J_{2} \stackrel{K}{=} L_{1}\left(m_{2}-1\right)$.

Let us show that functions (10) are upper solutions of problem (1)-(3). According to the comparison principle of solutions [1, pp.21-22], they must satisfy the following system of inequalities

$$
\begin{gathered}
\left\{\begin{array}{l}
\frac{\partial \widehat{u}}{\partial t} \geqslant \frac{\partial}{\partial x}\left(\widehat{v}^{m_{1}-1} \frac{\partial \widehat{u}}{\partial x}\right), \quad x>0, \quad t>0, \\
\frac{\partial \widehat{v}}{\partial t} \geqslant \frac{\partial}{\partial x}\left(\widehat{u}^{m_{2}-1} \frac{\partial \widehat{v}}{\partial x}\right), \quad x>0, \quad t>0,
\end{array}\right. \\
\left\{\begin{array}{l}
-\left.\widehat{v}^{m_{1}-1} \frac{\partial \widehat{u}}{\partial x}\right|_{x=0} \geqslant \widehat{u}^{q_{1}}(0, t), \quad t>0, \\
-\left.\widehat{u}^{m_{2}-1} \frac{\partial \widehat{v}}{\partial x}\right|_{x=0} \geqslant \widehat{v}^{q_{2}}(0, t), \quad t>0 .
\end{array}\right.
\end{gathered}
$$

Taking into account that

$$
\begin{gathered}
\widehat{u}_{t}=L_{1} e^{L_{1} t}\left(K+e^{-M_{1} \xi_{1}}\right)+M_{1} J_{1} x e^{\left(L_{1}-J_{1}\right) t} \geqslant L_{1} e^{L_{1} t}(K+1), \\
\widehat{v}^{m_{1}-1} \widehat{u}_{x}=M_{1} e^{\left(L_{2}\left(m_{1}-1\right)+L_{1}-J_{1}\right) t}\left(K+e^{-M_{2} \xi_{2}}\right)^{m_{1}-1} e^{-M_{1} \xi_{1}}, \\
\left(\widehat{v}^{m_{1}-1} \widehat{u}_{x}\right)_{x}=M_{1} e^{\left(L_{2}\left(m_{1}-1\right)+L_{1}-J_{1}\right) t}\left(K+e^{-M_{2} \xi_{2}}\right)^{m_{1}-2} \times \\
\times\left(M_{1} e^{-\left(M_{1} \xi_{1}+J_{1} t\right)}\left(K+e^{-M_{2} \xi_{2}}\right)+\left(m_{1}-1\right) M_{2} e^{-J_{2} t}\right) \leqslant \\
\leqslant M_{1} e^{\left(L_{2}\left(m_{1}-1\right)+L_{1}-J_{1}\right) t}(K+1)^{m_{1}-2}\left(M_{1}(K+1)+\left(m_{1}-1\right) M_{2}\right), \\
\widehat{v}_{t}=L_{2} e^{L_{2} t}\left(K+e^{-M_{2} \xi_{2}}\right)+M_{2} J_{2} x e^{\left(L_{2}-J_{2}\right) t} \geqslant L_{2} e^{L_{2} t}(K+1), \\
\widehat{u}^{m_{2}-1} \widehat{v}_{x}=M_{2} e^{\left(L_{1}\left(m_{2}-1\right)+L_{2}-J_{2}\right) t}\left(K+e^{-M_{1} \xi_{1}}\right)^{m_{2}-1} e^{-M_{2} \xi_{2}}, \\
-616-
\end{gathered}
$$




$$
\begin{aligned}
\left(\widehat{u}^{m_{2}-1} \widehat{v}_{x}\right)_{x} & =M_{2} e^{\left(L_{1}\left(m_{2}-1\right)+L_{2}-J_{2}\right) t}\left(K+e^{-M_{1} \xi_{1}}\right)^{m_{2}-2} \times \\
& \times\left(M_{2} e^{-\left(M_{2} \xi_{2}+J_{2} t\right)}\left(K+e^{-M_{1} \xi_{1}}\right)+\left(m_{2}-1\right) M_{1} e^{-J_{1} t}\right) \leqslant \\
& \leqslant M_{2} e^{\left(L_{1}\left(m_{2}-1\right)+L_{2}-J_{2}\right) t}(K+1)^{m_{2}-2}\left(M_{2}(K+1)+\left(m_{2}-1\right) M_{1}\right)
\end{aligned}
$$

and considering definitions of $M_{i}, J_{i}, L_{i}(i=1,2)$ and $K$, it is easy to prove that at $q_{1} \leqslant 1, q_{2} \leqslant 1$ systems of inequalities (11) and (12) are always true.

Theorem 2.2. Let $q_{1}>1, q_{2}>1$. Then each solution of problem (1)-(3) is unbounded at sufficiently large initial data.

Proof. To prove Theorem 2.2 the solution of problem (1)-(3) is taken in the following selfsimilar form

$$
\left\{\begin{array}{l}
\underline{u}(x, t)=(T-t)^{-\alpha_{1}} \varphi(\xi), \\
\underline{v}(x, t)=(T-t)^{-\alpha_{2}} \phi(\xi), \xi=x(T-t)^{-\beta},
\end{array}\right.
$$

where $T>0, \beta=\frac{q_{1}-m_{2}}{2 q_{1}-m_{2}-1}=\frac{q_{2}-m_{1}}{2 q_{2}-m_{1}-1}, \alpha_{1}=\frac{1}{2 q_{1}-m_{2}-1}, \alpha_{2}=\frac{1}{2 q_{2}-m_{1}-1}$. Functions $(\varphi(\xi), \phi(\xi))$ are solutions of the following problems

$$
\begin{gathered}
\left\{\begin{array}{l}
\frac{d}{d \xi}\left(\phi^{m_{1}-1} \frac{d \varphi}{d \xi}\right)-\beta \xi \frac{d \varphi}{d \xi}-\alpha_{1} \varphi=0 \\
\frac{d}{d \xi}\left(\varphi^{m_{2}-1} \frac{d \phi}{d \xi}\right)-\beta \xi \frac{d \phi}{d \xi}-\alpha_{2} \phi=0
\end{array}\right. \\
\left\{\begin{array}{l}
-\phi^{m_{1}-1} \frac{d \varphi}{d \xi}(0)=\varphi^{q_{1}}(0) \\
-\varphi^{m_{2}-1} \frac{d \phi}{d \xi}(0)=\phi^{q_{2}}(0)
\end{array}\right.
\end{gathered}
$$

which are obtained after substituting (13) into (1)-(3) and some simplifications. Conditions can be attained wherein solution (13) is an unbounded lower solution of problem (1)-(3). The following functions are compared

$$
\left\{\begin{array}{l}
\tilde{\varphi}(\xi)=A_{1}(a-\xi)^{\frac{1}{m_{2}-1}} \\
\tilde{\phi}(\xi)=A_{2}(a-\xi)^{\frac{1}{m_{1}-1}}
\end{array}\right.
$$

where $A_{i}>0(i=1,2)$. Then in order to use the comparison theorem the following inequalities should be satisfied

$$
\begin{gathered}
\left\{\begin{array}{l}
\frac{d}{d \xi}\left(\tilde{\phi}^{m_{1}-1} \frac{d \tilde{\varphi}}{d \xi}\right)-\beta \xi \frac{d \tilde{\varphi}}{d \xi}-\alpha_{1} \tilde{\varphi} \geqslant 0 \\
\frac{d}{d \xi}\left(\tilde{\varphi}^{m_{2}-1} \frac{d \tilde{\phi}}{d \xi}\right)-\beta \xi \frac{d \tilde{\phi}}{d \xi}-\alpha_{2} \tilde{\phi} \geqslant 0
\end{array}\right. \\
\left\{\begin{array}{l}
-\tilde{\phi}^{m_{1}-1} \frac{d \tilde{\varphi}}{d \xi}(0) \leqslant \tilde{\varphi}^{q_{1}}(0) \\
-\tilde{\varphi}^{m_{2}-1} \frac{d \tilde{\phi}}{d \xi}(0) \leqslant \tilde{\phi}^{q_{2}}(0)
\end{array}\right.
\end{gathered}
$$

This is equivalent to stating that the following inequalities

$$
\left\{\begin{array}{l}
\frac{A_{2}^{m_{1}-1}}{\left(m_{1}-1\right)^{2}}-\alpha_{1} a+\left(\alpha_{1}+\frac{\beta_{1}}{m_{2}-1}\right) \xi \geqslant 0, \\
\frac{A_{1}^{m_{2}-1}}{\left(m_{2}-1\right)^{2}}-\alpha_{2} a+\left(\alpha_{2}+\frac{\beta_{2}}{m_{1}-1}\right) \xi \geqslant 0,
\end{array}\right.
$$




$$
\left\{\begin{array}{l}
A_{1} A_{2}^{m_{1}-1} a^{\frac{1}{m_{2}-1}} \leqslant A_{1}^{q_{1}} a^{\frac{q_{1}}{m_{2}-1}} \\
A_{2} A_{1}^{m_{2}-1} a^{\frac{1}{m_{1}-1}} \leqslant A_{2}^{q_{2}} a^{\frac{q_{2}}{m_{1}-1}}
\end{array}\right.
$$

are true. It is clear that the first systems of inequalities are true if the following sufficient conditions

$$
a \leqslant \min \left\{\frac{A_{1}^{m_{1}-1}}{\alpha_{2}\left(m_{1}-1\right)^{2}}, \frac{A_{2}^{m_{2}-1}}{\alpha_{1}\left(m_{2}-1\right)^{2}}\right\}, \quad q_{1}>1, \quad q_{2}>1
$$

are satisfied.

From the second systems of inequalities the following restrictions on $a$ are obtained

$$
a \geqslant \max \left\{\frac{A_{1}^{\frac{\left(m_{1}-1\right)\left(m_{2}-1\right)}{q_{2}-1}}}{\left(m_{1}-1\right)^{\frac{m_{1}-1}{q_{2}-1}} A_{2}^{m_{1}-1}}, \frac{A_{2}^{\frac{\left(m_{1}-1\right)\left(m_{2}-1\right)}{q_{1}-1}}}{\left(m_{2}-1\right)^{\frac{m_{2}-1}{q_{1}-1}} A_{1}^{m_{2}-1}}\right\} .
$$

Then, choosing values of $A_{1}, A_{2}, a$, we can always achieve that the last system of inequalities are satisfied at $q_{1}>1, q_{2}>1$. According to the comparison principle of solutions, the lower self-similar solutions (13), (16) proved conditions for the initial data:

$$
\left\{\begin{array}{l}
u_{0}(x) \geqslant T^{-\alpha_{1}} A_{1}\left(a-x^{2} T^{-2 \beta}\right)_{+}^{\frac{1}{m_{2}-1}} \\
v_{0}(x) \geqslant T^{-\alpha_{2}} A_{2}\left(a-x^{2} T^{-2 \beta}\right)_{+}^{\frac{1}{m_{1}-1}}
\end{array}\right.
$$

Thus, by comparison we conclude that

$$
\left\{\begin{array}{l}
u(x, t) \geqslant(T-t)^{-\alpha_{1}} \tilde{\varphi}(0) \rightarrow \infty, t \rightarrow T, \\
v(x, t) \geqslant(T-t)^{-\alpha_{2}} \tilde{\phi}(0) \rightarrow \infty, t \rightarrow T,
\end{array}\right.
$$

at $q_{1}>1$ and $q_{2}>1$. It implies that $u$ and $v$ are unbounded. The theorem is proved.

Theorem 2.3. Suppose that $q_{1}>m_{2}+1, q_{2}>m_{1}+1$ and initial data are sufficiently small. Then each solution of problem (1)-(3) is global.

Proof. Conditions for the solvability in time of problem (1)-(3) are determined by constructing bounded upper solutions. They are taken in the following self-similar form:

$$
\left\{\begin{array}{l}
u_{+}(x, t)=(T+t)^{-\alpha_{1}} f(\xi) \\
v_{+}(x, t)=(T+t)^{-\alpha_{2}} g(\xi), \quad \xi=x(T+t)^{-\beta},
\end{array}\right.
$$

where $T>0$. Taking into account the comparison theorem of solutions, functions $(f(\xi), g(\xi))$ should satisfy the system of inequalities

$$
\begin{gathered}
\left\{\begin{array}{c}
\frac{d}{d \xi}\left(g^{m_{1}-1} \frac{d f}{d \xi}\right)+\beta \xi \frac{d f}{d \xi}+\alpha_{1} f \leqslant 0 \\
\frac{d}{d \xi}\left(f^{m_{2}-1} \frac{d g}{d \xi}\right)+\beta \xi \frac{d g}{d \xi}+\alpha_{2} g \leqslant 0
\end{array}\right. \\
\left\{\begin{array}{l}
-g^{m_{1}-1} \frac{d f}{d \xi}(0) \geqslant f^{q_{1}}(0), \\
-f^{m_{2}-1} \frac{d g}{d \xi}(0) \geqslant g^{q_{2}}(0) . \\
-618-
\end{array}\right.
\end{gathered}
$$


Let us consider the following functions

$$
\left\{\begin{array}{l}
\bar{f}(\xi)=A_{1}\left(a-\left(\xi+h_{1}\right)^{2}\right)^{\frac{1}{m_{2}-1}} \\
\bar{g}(\xi)=A_{2}\left(a-\left(\xi+h_{2}\right)^{2}\right)^{\frac{1}{m_{1}-1}}
\end{array}\right.
$$

where $h_{1}, h_{2} \in(0, \sqrt{a}), a>0, A_{1}=\left(\frac{\beta\left(m_{1}-1\right)}{2}\right)^{\frac{1}{m_{2}-1}}, A_{2}=\left(\frac{\beta\left(m_{2}-1\right)}{2}\right)^{\frac{1}{m_{1}-1}}$. Systems of inequalities (18), (19) are solvable with respect to unknowns $a, h_{1}$ and $h_{2}$ at $q_{1}>m_{2}+1$, $q_{2}>m_{1}+1$. Then, substituting functions (20) in (18) and (19), we obtain

$$
\left\{\begin{array}{c}
\left(\alpha_{1}-\beta\right)\left(a-\left(\xi+h_{1}\right)^{2}\right) \leqslant 0 \\
\left(\alpha_{2}-\beta\right)\left(a-\left(\xi+h_{2}\right)^{2}\right) \leqslant 0
\end{array}\right.
$$

Then necessary conditions $q_{1}>m_{2}+1, q_{2}>m_{1}+1$ and conditions for numerical parameters $a$, $h_{1}, h_{2}$

$$
a \leqslant \min \left\{h_{1}^{2}+\left(A_{2}^{m_{1}-1} A_{1}^{1-q_{1}} \frac{2 h_{1}}{m_{2}-1}\right)^{\frac{1}{q_{1}+m_{2}-2}}, h_{2}^{2}+\left(A_{1}^{m_{2}-1} A_{2}^{1-q_{2}} \frac{2 h_{2}}{m_{1}-1}\right)^{\frac{1}{q_{2}+m_{1}-2}}\right\}
$$

are obtained. Thus, if $q_{1}>m_{2}+1, q_{2}>m_{1}+1$, and initial functions $u_{0}(x), v_{0}(x)$ satisfy conditions

$$
\left\{\begin{array}{l}
u_{0}(x) \leqslant T^{-\alpha_{1}} A_{1}\left(a-\left(x T^{-\beta}+h_{1}\right)^{2}\right)^{\frac{1}{m_{2}-1}} \\
v_{0}(x) \leqslant T^{-\alpha_{2}} A_{2}\left(a-\left(x T^{-\beta}+h_{2}\right)^{2}\right)^{\frac{1}{m_{1}-1}}
\end{array}\right.
$$

where $a, h_{1}, h_{2}$ are chosen from condition (21), then the solution of problem (1)-(3) is global.

Remark 1. Theorem 2.1 shows that critical exponents of global existence of solution are $q_{10}=1, q_{20}=1$.

Remark 2. Theorem 2.3 shows that critical exponents of Fujita type are $q_{1 c}=m_{2}+1$, $q_{2 c}=m_{1}+1$.

\section{References}

[1] Z.Q.Wu, J.N.Zhao, J.X.Yin, H.L.Li, Nonlinear Diffusion Equations, Singapore, World Scientific, 2001.

[2] M.M.Aripov, Methods of Reference Equations for Solving Nonlinear Boundary Value Problems, Tashkent, Fan, 1988.

[3] A.S.Kalashnikov, Some Questions of the Qualitative Theory of Nonlinear Degenerate Second-order Parabolic Equations, Uspekhi Mat. Nauk, 42(1987), no. 2 (254), 135-176.

[4] J.D.Murray, Mathematical Biology, 3rd ed., Berlin, Springer, 2002,.

[5] H.Malchow, S.V.Petrovskii, E.Venturino, Spatiotemporal Patterns in Ecology and Epidemiology: Theory, Models, and Simulations, London, Chapman \& Hall/CRC Press, 2008. 
[6] M.A.Tsyganov, V.N.Biktashev, J.Brindley, A.V.Holden, G.R.Ivanitsky, Waves in Crossdiffusion Systems - a Special Class of Nonlinear Waves, UFN, 177(2007), no. 3, 275-300.

[7] H.Levine, The Role of Critical Exponents in Blowup Theorems, SIAM Rev., 32(1990), no. 2, $262-288$.

[8] S.Wang, C.H.Xie, M.X.Wang, Note on Critical Exponents for a System of Heat Equations Coupled in the Boundary Conditions, J Math Analysis Applic, 218(1998), 313-324.

[9] S.Wang, C.H.Xie, M.X.Wang, The Blow-up Rate for a System of Heat Equations Completely Coupled in the Boundary Conditions, Nonlinear Anal, 35(1999), 389-398.

[10] F.Quiros, J.D.Rossi, Blow-up Set and Fujita-type Curves for a Degenerate Parabolic System with Nonlinear Conditions, Indiana Univ Math J, 50(2001), 629-654.

[11] S.N.Zheng, X.F.Song, Z.X.Jiang, Critical Fujita Exponents for Degenerate Parabolic Equations Coupled Via Nonlinear Boundary Flux, J. Math. Anal. Appl., 298(2004), 308-324.

[12] Z.Rakhmonov, On the Properties of Solutions of Multidimensional Nonlinear Filtration Problem with Variable Density and Nonlocal Boundary Condition in the Case of Fast Diffusion, J. Sib. Fed. Univ. Math. \& Phys., 9(2016), no. 2, 236-245.

[13] Z.Rakhmonov, Assessment for the Solutions of a Nonlinear System of Heat Conduction Equations with Variable Density and Nonlocal Boundary Condition, Bulletin NUU, 2016, no. 1 (2), 145-154.

[14] M.M.Aripov, A.S.Matyakubov, To the Qualitative Properties of Solution of System Equations not in Divergence Form of Polytrophic Filtration in Variable Density, Nanosystems: Physics, Chemistry, Mathematics, 8(2017), no. 3, 317-322.

[15] M.M.Aripov, A.S.Matyakubov, Self-similar Solutions of a Cross-diffusion Parabolic System with Variable Density: Explicit Estimates and Asymptotic Behavior. Nanosystems: Physics, Chemistry, Mathematics, 8(2017), no. 1, 5-12.

[16] M.M.Aripov, Z.R.Rakhmonov, To the asymptotics of the solutions of a nonlinear heat conduction problem with a gradient nonlinearity, Uzbek Math. J., (2013), no. 3. 19-27.

\section{Об одной задаче кросс-диффузии с нелокальными граничными условиями}

Зафар Р. Рахмонов

Национальный университет Узбекистана Университет, 4, 100174

Узбекистан

Жасур Э. Урунбаев

Самаркандский государственный университет Университетский бул., 15, Самарканд, 140104

Узбекистан

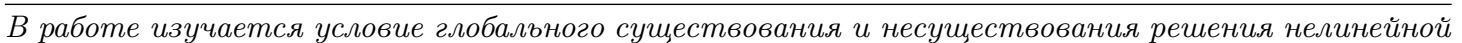
системы кросс-дифбузии с нелинейными граничными условиями. Установлены критические экспоненты типа Фуджита и критические экспоненты для глобального существования решения.

Ключевые слова: нелинейная параболическая система, кросс-дифбузия, критические экспонентъ типа Фуджита, режим с обострением. 